\title{
Surface Ultrastructure of Antenna and Distribution of Sensilla in the Leptogenys chinensis (Mayr)(Fabricus) (Formicidae: Hymenoptera)
}

\author{
Deepak D. Barsagade, Dewashree A. Nagarkar*, Jitendra R. Kirsan \\ Department of Zoology, MJF Educational campus, RTM Nagpur University, Nagpur-440033, Maharashtra, India.
}

\begin{tabular}{|c|c|}
\hline ARTICLE INFO & ABSTRACT \\
\hline Article history: & \multirow{5}{*}{$\begin{array}{l}\text { Ants have a well-developed pair of antenna with sensory system used in chemical communication for social } \\
\text { lives. The antennae comprise of two parts scape and funiculus. Funiculus contains single segmented pedicel } \\
\text { and ten segmented flagellum. The surface micro-morphology of the antennal sensilla of Leptogenys chinensis } \\
\text { worker shows the presence of various types of sensilla viz. sensilla trichodea, sensilla trichodea curvata, sensilla } \\
\text { basiconica, sensilla coeloconica, sensilla chaetica, sensilla coelocapitular. Sensilla trichodea is distributed on all } \\
\text { parts of the antenna. Sensilla trichodea curvata type III is typically present on flagellar segments only. Sensilla } \\
\text { basiconica with arrowhead present on scape ball is slightly different than the sensilla basiconica with rounded } \\
\text { head present on the flagellar tip. Sensilla coelocapitular is observed only on the flagellar tip. On the joint of scape } \\
\text { ball and pedicilar shaft, three types of sensilla trichodea are present on the ball ridge while sensilla basiconica } \\
\text { distributed on the dorso-lateral margin of the ball.. }\end{array}$} \\
\hline Received on: May 14,2018 & \\
\hline Accepted on: June 19, 2018 & \\
\hline Available online: March 05. 2019 & \\
\hline $\begin{array}{l}\text { Key words: Ant, Leptogenys, } \\
\text { antenna, sensilla, SEM. }\end{array}$ & \\
\hline
\end{tabular}

\section{INTRODUCTION}

On insect antennae, there are various kinds of sensillum with different functions that have important roles throughout the life history of the insect [1]. Parasitic hymenopterans have specialized sensory organs, such as habitat searching, host localization, recognition, selection and acceptance, courtship, mating and oviposition [2]. The chemosensory sensilla provide information related to humidity, temperature and $\mathrm{CO}_{2}$ levels about the surrounding area apart from the chemoreceptor [3,4,5].

There is a great deal of recent interest in how the sensory capabilities of an animal are reflected in the structural organization of its brain [6]. The antennae are complex sensory arrays studded with different types of sensilla which process a range of inputs in appearance despite a common underlying architecture [7].

Hymenopteran insect antenna contains various sensilla with variation in a different caste, observed under electron microscope $[8,9,10]$.

The morphology of the external cuticular element is, at least in part, dictated by the function of the particular sensillum. The chemoreceptors with pores, slits or other inlets allow molecules to penetrate into the lumen of sensillum. This permits different types of sensilla to be identified to a certain degree, based on external morphology alone

*Corresponding Author

Dewashree A. Nagarkar, New English Junior College, Mahal,

Nagpur-440032, Maharashtra, India.

E-mail: dewashreenagarkar@gmail.com
[11]. Despite the heavy reliance of ants on their antennae, we know relatively little about sensilla, their distribution, and abundance.

The present SEM studies of the antenna were carried out to know the various types of sensilla present on the worker of Leptogenys chinensis.

\section{MATERIAL AND METHODS}

Worker ants were collected from a single colony located in the vicinity of MJF education campus RTM Nagpur University, Nagpur, India. The worker ants used for scanning electron microscopy (SEM) analysis were immobilized \& antennae were removed. Material fixed in $70 \%$ alcohol for $12 \mathrm{~h}$ then dehydrated in ethanol, cleared in acetone and air dried. After that, the antenna was fixed on metallic stub at different angles and gold-coated separately. The samples were placed in the chamber specimens observed at a desirable magnification under a Jeol scanning electron microscope (JSM 6380A) at the SEM centre of Visvesvaraya National Institute of Technology, Nagpur, India.

\section{RESULTS AND DISCUSSION}

The antenna of the workers of Leptogenys chinensis divided into scape and funiculus whereas funiculus is subdivided into pedicel and flagellum. Flagellum consists 10 flagellar segments while scape and pedicel are single segmented (Figure 1). On the dorso-lateral side of the head, the antenna is a wedge in the antennal socket. Flagellum folded up against scape, representing the elbowed geniculate type of antenna measured about $5.9 \mathrm{~mm}$ in length (Table 1). The surface micro- 
morphology of antennal sensilla of $L$. chinensis shows the presence of various types of sensilla (Table 2).

Table 1: Morphology of worker antenna Leptogenys chinensis. (Values are mean \pm standard deviation).

\begin{tabular}{ccc} 
STRUCTURE & $\begin{array}{c}\text { SIZE (mm) } \\
\text { WORKER }\end{array}$ & Width \\
Antennal socket (D) & $0.20 \pm 0.011$ & \\
Socket ring (T) & $0.0038 \pm 0.00021$ & \\
Ball Shaft (joint between ball and shaft) & $0.102 \pm 0.006$ & \\
Scape (L) & $2.02 \pm 0.29$ & $0.316 \pm 0.002$ \\
Pedicel (L) & $0.372 \pm 0.014$ & $0.182 \pm 0.0014$ \\
Flagellum F1-F10 (L) & $3.16 \pm 0.25$ & $0.272 \pm 0.0011$ \\
Antenna (TL) & $5.9 \pm 0.42$ & \\
\hline
\end{tabular}

D - Diameter, T - Thickness, L - Length, TL - Total Length.

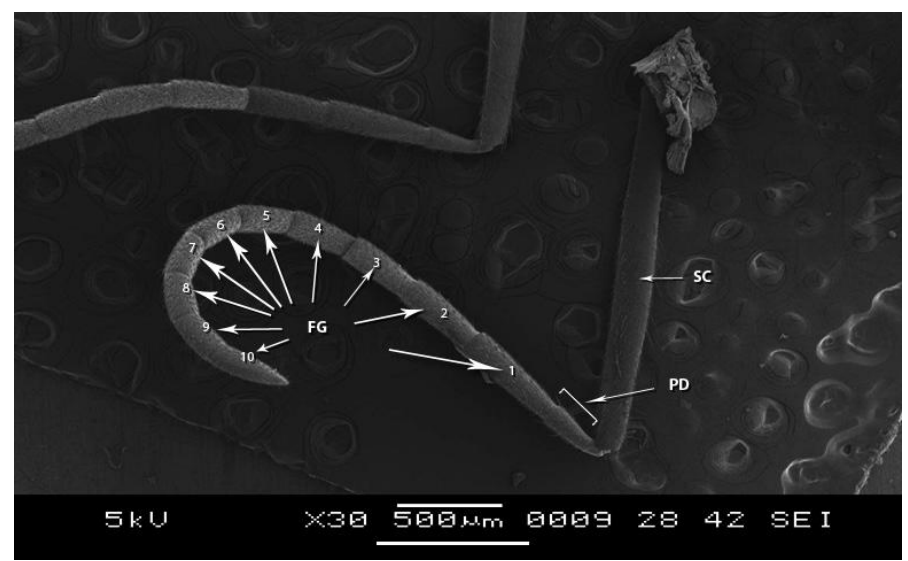

Figure 1: SEM structure of antenna of worker ant. SC - Scape, PD - Pedical, FG - Flagellum.

\subsection{Results}

\subsubsection{Sensilla present on scape}

The scape is subdivided into three subparts as scape ball, ball neck and elongated shaft. On scape ball, three types of basiconic sensilla, sensilla basiconica III (SB-III), sensilla basiconica II (SB-II) and sensilla basiconica I (SB-I) were observed on dorso-lateral side (Figures 2-4). The SB-I was smooth while, SB-II was smaller than SB-I and SB-III is one of the shortest sensilla present on scape ball (Table 2) (Figure 5).

\subsubsection{Sensilla present on ball neck}

Small elongated region known as ball neck present in between the scape ball and the elongated shaft of scape (Figure 3). On dorsal side, along the ridge only, three types of trichodea sensilla were observed ST-I, ST-II and ST-III (Figure 5). ST-I were longer among three and ST-III were shortest (Table 2). These sensilla were pointed terminally. In contrast to other trichoid sensilla that are angles towards the tip of antenna, these sensilla project almost perpendicularly from antennal surface (Figure 5).

\subsubsection{Sensilla present on elongated shaft of scape}

On the entire surface of scape three type of sensilla trichodea and two types of sensilla trichodea curvata (Table 2) are present (Figure 6). Sensilla basiconic were also seen on the terminal region towards the pedicel, number is less. Sensilla coeloconica with external opening were observed on the ventral side of scape (Figure 7).

Table 2: Distribution and size of sensilla on different segments of the antenna on worker of Leptogenys chinensis. Given are the measurements of the organs (mean + standard deviation).

\begin{tabular}{|c|c|c|c|c|}
\hline \multirow{2}{*}{\multicolumn{2}{|c|}{ Parts of the antenna }} & \multirow[t]{2}{*}{ Type } & \multicolumn{2}{|c|}{ Size $(\mu \mathrm{m})$} \\
\hline & & & $\mathbf{L}$ & W \\
\hline \multirow{6}{*}{\multicolumn{2}{|c|}{$\begin{array}{c}\text { Ball Neck } \\
\text { (joint ball and shaft) }\end{array}$}} & SB-I & $24.49 \pm 3.64$ & $4.07 \pm 0.12$ \\
\hline & & SB-II & $9.018 \pm 1.60$ & $2.45 \pm 0.21$ \\
\hline & & SB-III & $4.31 \pm 1.38$ & $1.4 \pm 0.14$ \\
\hline & & ST-I & $62.29 \pm 2.16$ & $3.2 \pm 0.41$ \\
\hline & & ST-II & $47.07 \pm 3.31$ & $2.5 \pm 0.24$ \\
\hline & & ST-III & $34.62 \pm 2.16$ & $1.60 \pm 0.24$ \\
\hline \multirow[t]{13}{*}{ Scape } & \multirow{7}{*}{ Shaft } & ST-I & $167.03 \pm 17.41$ & $5 \pm 0.707$ \\
\hline & & ST-II & $71.33 \pm 5.78$ & $3.41 \pm 0.26$ \\
\hline & & ST-III & $40.94 \pm 5.06$ & $2.6 \pm 0.14$ \\
\hline & & STC-I & $48.89 \pm 2.46$ & $5.35 \pm 0.35$ \\
\hline & & STC-II & $30.14 \pm 7.79$ & $2.24 \pm 0.36$ \\
\hline & & SB & $6.78 \pm 2.39$ & $2.4 \pm 0.14$ \\
\hline & & $\mathrm{SC}(\mathrm{D})$ & $3.31 \pm 1.41$ & -- \\
\hline & \multirow{6}{*}{ Pedicel } & ST-I & $144.1 \pm 1.5$ & $3.79 \pm 0.15$ \\
\hline & & ST-II & $96.6 \pm 2.02$ & $2.6 \pm 0.42$ \\
\hline & & ST-III & $35.78 \pm 4.99$ & $1.45 \pm 0.16$ \\
\hline & & STC-I & $52.5 \pm 3.32$ & $2.16 \pm 0.11$ \\
\hline & & STC-II & $32.43 \pm 1.7$ & $1.13 \pm 0.15$ \\
\hline & & $\mathrm{SCh}$ & $26.36 \pm 1.28$ & $1.07 \pm 0.03$ \\
\hline \multirow{10}{*}{ Funiculus } & \multirow{10}{*}{ Flagellum } & ST-I & $48 \pm 2.44$ & $4.27 \pm 0.19$ \\
\hline & & ST-II & $33.3 \pm 4.5$ & $1.95 \pm 0.12$ \\
\hline & & ST-III & $17.26 \pm 0.74$ & $0.85 \pm 0.07$ \\
\hline & & STC-I & $16.95 \pm 0.72$ & $2.26 \pm 0.03$ \\
\hline & & STC-II & $7.97 \pm 0.86$ & $1.45 \pm 0.03$ \\
\hline & & STC-III & $19.70 \pm 1.02$ & $10.59 \pm 0.81$ \\
\hline & & $\mathrm{SCh}$ & $18.62 \pm 1.72$ & $1.21 \pm 0.03$ \\
\hline & & SB & $21.66 \pm 1.44$ & $3.92 \pm 0.84$ \\
\hline & & SCP & $1.4 \pm 0.14$ & $0.95 \pm 0.07(\mathrm{~B})$ \\
\hline & & & $\begin{array}{c}0.21 \pm 0.014 \\
(\mathrm{NP})\end{array}$ & \\
\hline
\end{tabular}

L - Length, W - Width, D - Diameter, B - Breadth SB - Sensilla Basiconica, ST - Sensilla Trichodea, STC - sensilla trichodea curvata, SC - Sensilla coeloconica, SCh - sensilla chaetica, SCP - sensilla coelocapitular, NP - Nub-like-projection.

\subsubsection{Sensilla present on pedicel}

Throughout the dorsal and ventral surface of pedicel, sensilla trichodea and trichodea curvata were densely distributed. Sensilla chaetica were also seen on the lateral side of pedicel (Figure 8).

\subsubsection{Sensilla present on Flagellum}

Sensilla trichodea (ST) and sensilla trichodea curvata (STC) were present heavily on dorsal as well as ventral side of flagellar segments (Figure 9). Three types of sensilla trichodea (ST-III, ST-II, ST-I) were observed on flagellar segment and three types of sensilla trichodea curvata (STC-I, STC-II, STC-III) were also seen. Sensilla trichodea 
curvata III with large elliptical peg socket (L - 11.66 \pm 2.08 ; W $-3.81 \pm$ 0.39 ) were observed only on flagellar segment (Figure 10).

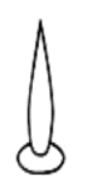

a) SB

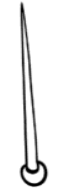

b) ST

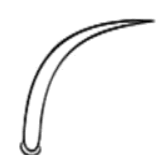

c) STC

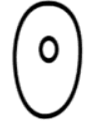

a) SCP

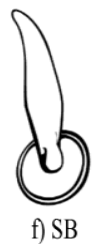

f) $\mathrm{SB}$

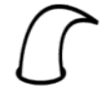

d) STC -III e) $\mathrm{SCh}$

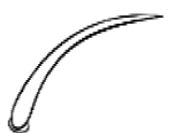

Figure 2: Schematic representation of different types of sensilla on the antenna SB - sensilla basiconica, ST - sensilla trichodea, STC - sensilla trichodea curvata, STC-III - sensilla trichodea curvata type III, SCh - sensilla chaetica, SCP - sensilla coelocapitular.

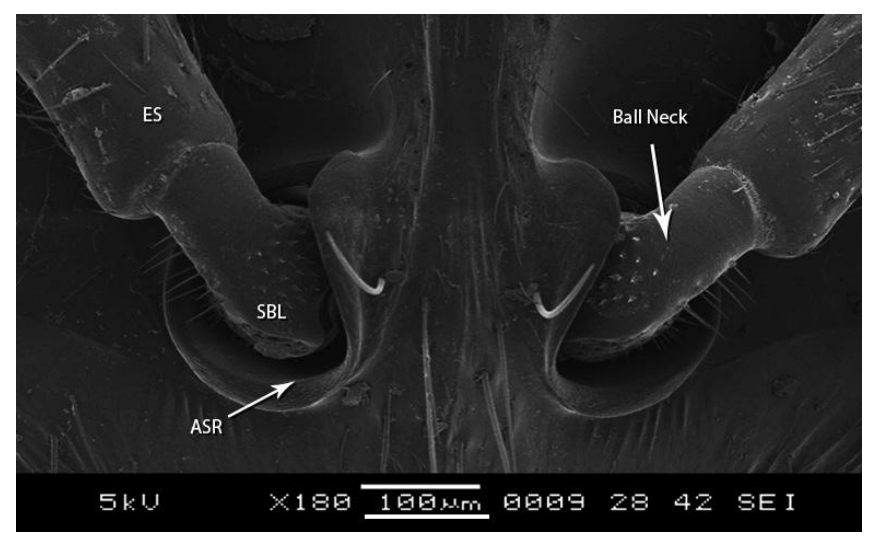

Figure 3: SEM structure of both antenna with a socket, ES - Elongated Shaft, SBL - Scape ball, ASR - Antennal Sclerotic ring.

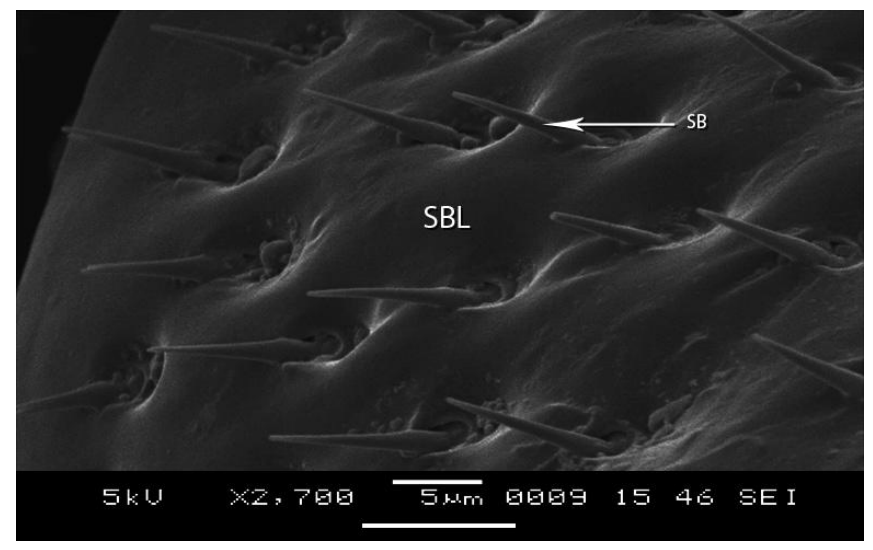

Figure 4: Magnified view of sensilla basiconica on scape ball.

Few number sensilla basiconica were also seen projecting perpendicularly on the antennal surface, structurally different from those SB observed on the scape. SB was with thickened pegs and rounded tip (Figure 9). SB was distributed in a form of a band slightly above the base of the $10^{\text {th }}$ flagellar segment. Slender hair like sensilla chaetica was also present on the flagellum.

Sensilla coelocapitular (SCP) were quite small with small nublike projections, surrounded by elliptical depression appears like peg socket. SCP was seen only around the tip of the apical flagellar segment (Figure 10).

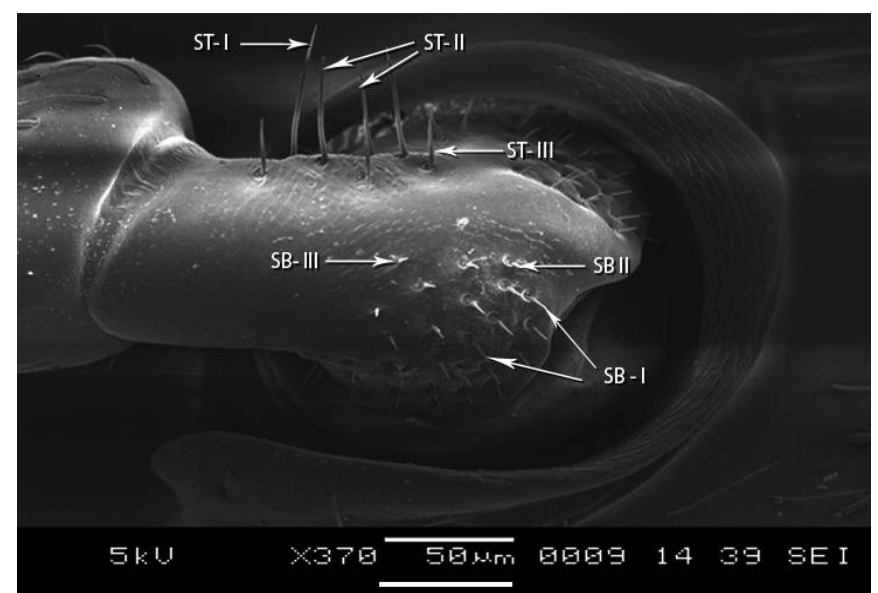

Figure 5: SEM structure of scape ball showing three types of sensilla basiconica, SB I, II, III and three types of sensilla trichodea.

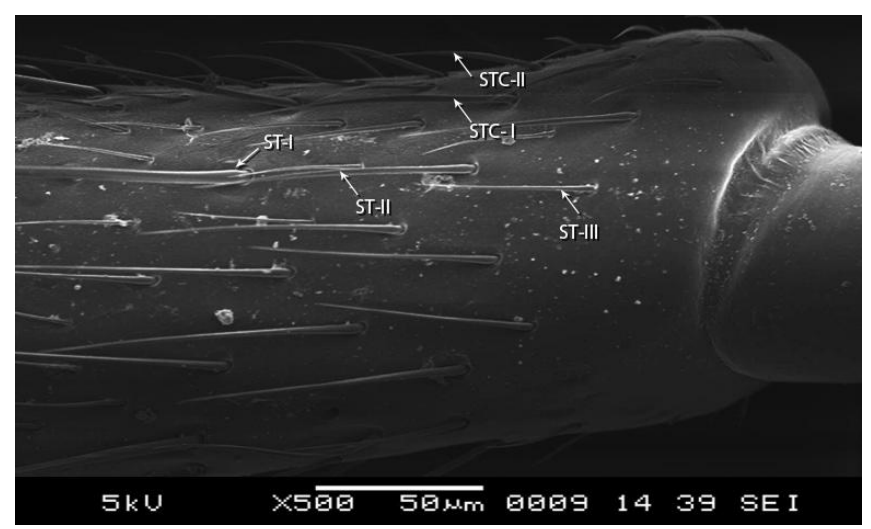

Figure 6: SEM structure of scape shaft showing ST-I, II, III, and sensilla trichodea curvata STC - I, II, III.

\subsection{Discussion}

The organization of antennal segments in the ant is similar to that in another well-known social Hymenoptera, the honeybee Apis mellifera and carpenter ant $C$. compressus a scape, a pedicle, and ten flagellar segments in the worker $[9,18,19]$. The geniculate type of antennae found in Leptogenys chinensis is typical of aculeate Hymenoptera $[6,11,12,13,14]$. The total number of flagellar segment i.e. 10 observed in the worker of $L$. chinensis is similar to that found in worker of $C$. compressus $[11,18,19,20]$.

In Diacamma sp. Antenna scape show ball-like structure (14) and C. compressus [18] and similar modification at the base of scape is evident in $L$. chinensis with ball and ball neck (a joint between ball and shaft).

A maximum number of flagellar sensilla in the ant is noted in Formicidae species $[15,16,17]$. In $L$. chinensis various sensilla are densely distributed on all parts of the antenna. Earlier various types of antennal sensilla have been noticed in the ants, Lasius fuliginosus (Latreille) [21], Diacamma sp. [4,14] and C. compressus [6,18]. The 
present study six types of antennal sensilla in L. chinensis, viz. sensilla trichodea (ST), senilla basiconica (SB), sensilla chaetica (SCh), sensilla trichodea curvata (STC), sensilla coelocapitular (SCP) and sensilla coeloconica (SC) in the worker, has been noticed.

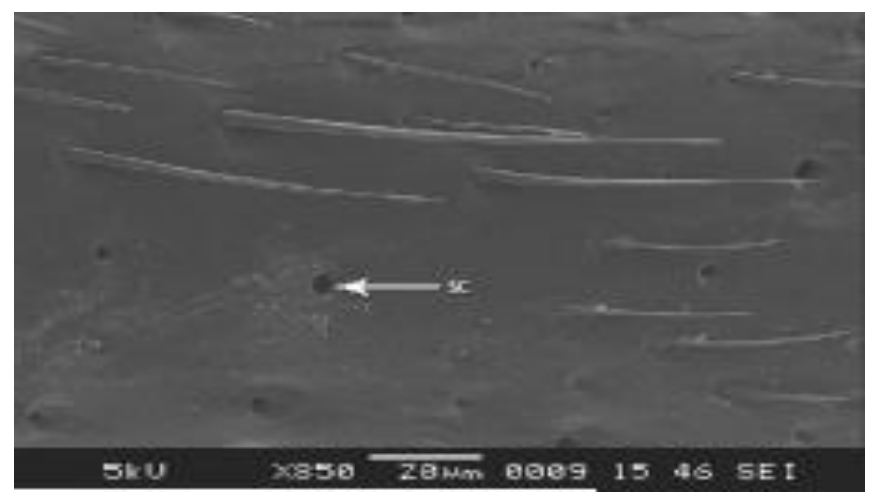

Figure 7: SEM structure of scape shaft showing sensilla coeloconica (SC).

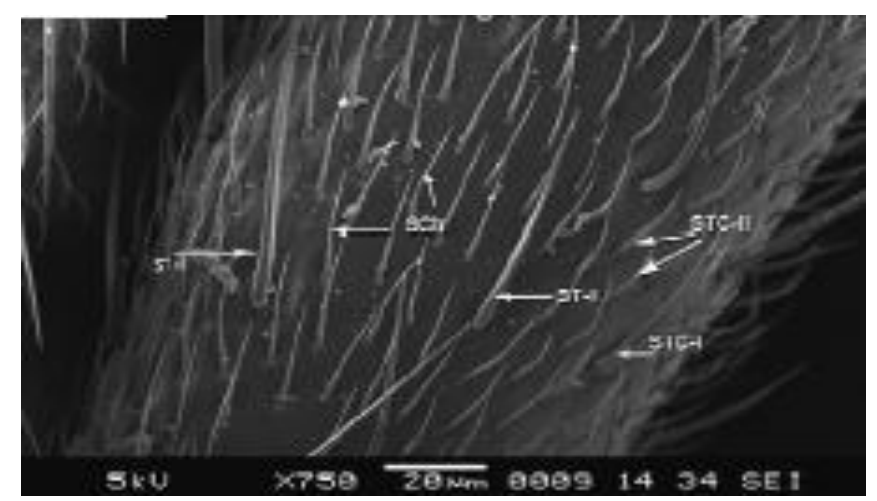

Figure 8: SEM structure of pedicel showing sensilla trichodea (ST), sensilla trichodea curvata (STC) and sensilla chaetica (SCh).

In Formicidae, antennal sensilla have been morphologically classified based on the appearances of their sensilla $[15,16,17,11]$. Seven types of flagellar sensilla are distinguished in Lasius fuliginosus: chaotic, trichoid, trichoid curvata, basiconic, coeloconic, ampullaceal and campaniform sensilla [15]. In the present study of L. chinensis, basiconic, trichoid, trichoid curvata, coeloconic, coelocapitular and chaetica sensilla have been observed on flagella. The coelocapitular sensillum on the flagella of $L$. chinensis is observed the first time and corresponds to the coelocapitular sensillum in C. japonicus [11] and honey bee [32].

The basiconic sensilla (SB) on scape ball of L. chinensis exhibits similar morphological structure SB present on C. compressus [18], Sclerodeermus sp. [22] and of M. pyriformis [7]. The socket of SB in $L$. chinensis is elevated above the level of the antennal surface, similar to that of Myrmeica gulosa [23]. Anatomical, electrophysiological and behavioral evidence indicates that sensilla basiconica function as contact gustatory chemoreceptors in Componotus vagus [24]; Componotus japonicas [4], might have a similar function of SB in $L$. chinensis.

According to Villa and Mineo [25,26], trichoid sensilla correspond to short structures, spine shaped, which occur on the ventral surface of the scape and pedicel base. Similar findings were also reported in $C$. compressus by Barsagade et al. [18]. In addition to this in L. chinensis sensilla trichodea were also seen on the ball neck between scape ball and elongated shaft. The trichoid sensilla occur in the antennal club are associated to the papillary while, chaetica type, in conjunction form a functional portion of the antenna defined as "touch and taste area". Similar finding evident in M. pyriformis and found ST associated with SB has the function of contact chemoreceptors [23,7]. In L. chinensis ST were found associated with sensilla chaetica and sensilla trichodea curvata on flagellum might function as contact chemosensory as noticed in invertebrates [33].

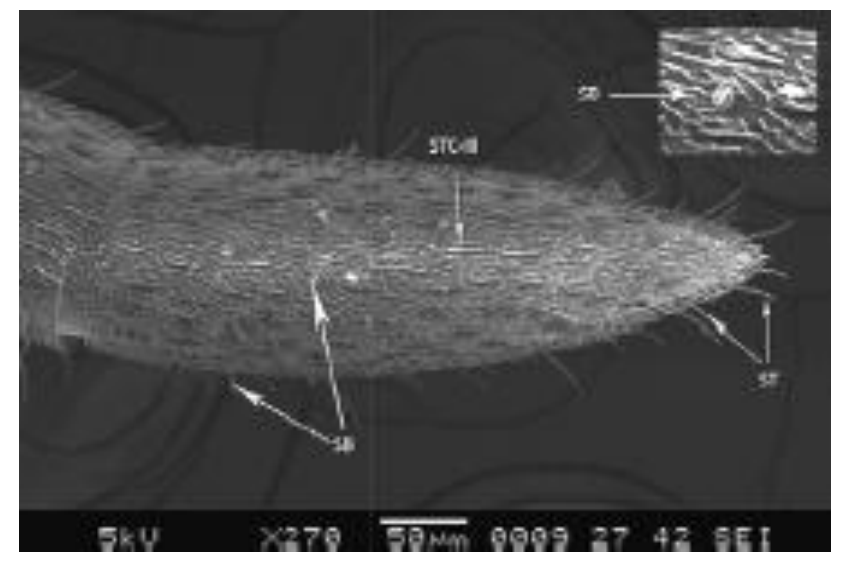

Figure 9: SEM structure of terminal flagellar segment, showing sensilla basiconica (SB) and STC-III sensilla trichodea curvata III, sensilla trichodea (ST), Inset showing sensilla basiconica.

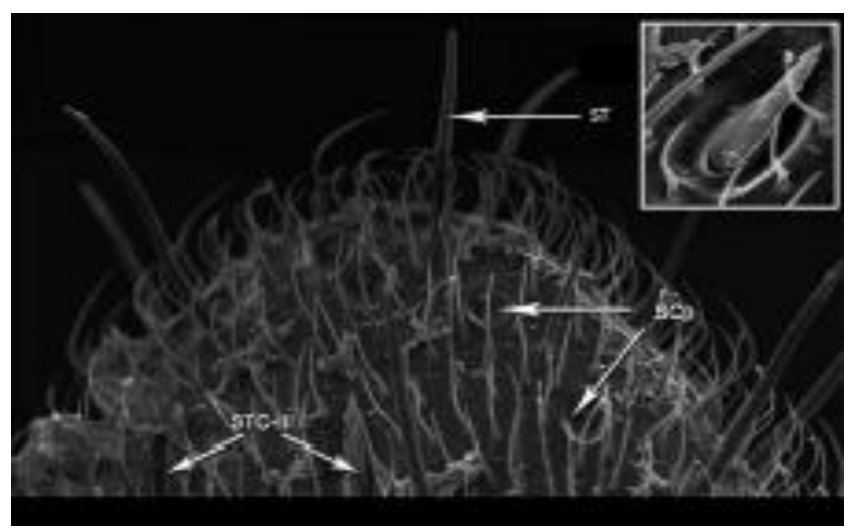

Figure 10: Magnified view of the tip of a terminal flagellar segment showing sensilla coelocapitular (SCp), sensilla trichodea (ST), sensilla trichodea curvata III (STC-III), Inset showing sensilla trichodea curvata III.

Rutchy et al. [27] measured sensory neuron activity and established that sensilla coeloconica respond to changes in the atmosphere. SC was reported to be observed only on flagomere in Sclerodermus sp. [22] and $C$. japonocus [11] on the contrary sensilla coeloconica were not observed in $C$. compressus [18]. In L. chinensis, sensilla coeloconica were observed on shaft region. The coeloconic sensillum work as chemoreceptor in red imported fire ant [17] and might be performing chemoreceptor function in L. chinensis.

The coelocapitular, coeloconic and ampullaceal sensilla are distributed on most flagellar segments in the ant, where as in the honeybee, they are not distributed on the first and second segments $[9,28]$. The coelocapitular sensilla are most densely distributed on the first flagellar segments in Componotus members. It is also present in C. japonicus [11], in honeybees [29] and in other insect orders such as Coleoptera and Mantophasmatodea [30,31]. 
Sensilla coelocapitular were also observed in L. chinensis on the flagellar tip. Earlier coelocapitular sensillum has been thoroughly studied in the honeybee, with electrophysiologically testing its neural connections to the glomeruli mapped; its anatomy has been described and confirms acting as both a hygro- and a thermoreceptor [28,32,29]. The presence of coelocapitular sensillum may play a similar role in $L$. chinensis perhaps this has the first report in L. chinensis.

Sensilla trichodea curvata type three observed on flagellum were similar to STC of nocturnal bull ant M. pyriformis [7]. STC III observed on flagellum is bilaterally flattened and strongly bend toward the antennal tip and they are most wide sensilla on the flagellum of $L$. chinensis. The location of the sensilla and the pore structure suggest that STC are olfactory sensilla for detection of volatile odors and pheromones [17] similar function might be assigned to STC in L. chinensis.

Sensilla trichodea located on the ball neck, in contrast to other trichoid sensilla on the antenna that are angled towards the tip of the antenna, these sensilla projects almost perpendicularly from the antennal surface, which makes them quite eye-catching. No sensilla yet reported to be located on ball neck region in ants. The trichoid sensillum observed in L. chinensis is probably the same type as the trichoid sensillum in other ant species working as olfactory sensilla $[11,15]$.

\section{CONCLUSION}

The various types of sensilla present on the antenna of $L$. chinensis are related to the humid terrestrial dwelling habitat and play important role in food searching capacity. This information provides basic morphological structure and may help to further behavioral study of ant.

\section{ACKNOLWEDMENT}

We are grateful to the Director, Visvesvaraya National Institute of Technology (VNIT) Nagpur, India for their help in SEM imaging. We are thankful to New English High school Association Nagpur, for their timely support.

\section{REFERENCES}

1. Schneider D. Insect antennae. Annual Review of Entomology 1964; 9:103-122.

2. Das P, Chen L, Sharma KR, Fadamiro HY. Abundance of antennal chemosensilla in two parasitoid wasps with different degree of host specificity may explain sexual and species differences in their response to host-related volatiles. Microscopy Research and Technology 2011; 74:900-909.

3. Fresneau D. Etude du rBle sensorial de l'antenne dam l'ethogenese des soins aux cocoons chez formica polyctena Forst. (Hymenoptera: formicidae). Insectes Sociux 1979; 26:170-195.

4. Ozaki M, Wada-Katsumata A, Fujikawa K, Iwahasi M, Yokahari F, Satiji Y, Nishimura T, Yamaoka Y. Ant nestmate and nonnestmate discrimination by a chemosensory sensillum. Science 2005; 309:311314.

5. Barsagade DD, Thakre RP, Gathalkar GB, Kirsan JR. A comparative study of antennal microstructure in two species of damselflies Rhodischnura nursei and Lestes elatus. Journal of Entamology and Zoology Studies 2017; 5(2):1550-1557.

6. Mysore K, Subramanium KA, Sarasij RC, Suresh A, Shyamala BV, Vijayraghvan K, Rodrigues V. Caste and sex specific olfactory glomerural organization and brain architecture in two sympatric ant species Componotus sericeus and Componotus compressus (Fabricius, 1798). Arthropod Structure and Development 2009; 38:485-497.
7. Ramirez-Esquivel F, Zeil J, Narendra A. The antennal sensory array of the nocturnal bull ant Myrmecia pyriformis. Elseveier 2014; 43(6):54358 .

8. Agren L, Svensson BG. Analysis of the antennal sensilla equipment of the bumble bee. Apidologie 1972; 13:247-64.

9. Esslen J, Kaissling KE. Zahl-und Verteilung antennaler sensillen beri der Honigbiene (Apis mellifera L.). Zoomorphology 1976; 83:227-251.

10. Agren L. Flagellar sensilla of some colletidae (Hymenoptera: Apoidea). International Journal of Insect Morphology and Embryology 1977; 4:137-146.

11. Nakanishi A, Nishino H, Watanabe H, Yokohari F, Nishikawa M. Sexspecific antennal sensory system in the ant Componotus japonicus: structure and distribution of sensilla on the flagellum. Cell Tissue Research 2009; 338:79-97.

12. Michener CD. 1974. The social behavior of the bees. Cambridge: Harvard University Press.

13. Richards OW, Davies RG. 1988. Classification and biology. In: Richards OW, Davies RG, ed. Imm's general textbook of Entamology $10^{\text {th }}$. Landon: Chapman and Hall, Vol. 1; p. 123-178.

14. Okada Y, Miura T, Tsuji K. Morphological differences between sexes in the ponerine ant, Diacamma sp. (Formicidae: Ponerinae). Sociobiology 2006; 48:527-541.

15. Dumpert K. Bau und Verteilung derSensillen auf der Antennengeissel von Lasius fuliginosus (Latr.) (Hymenoptera, Formicidae). Zeitschrift Morphologie der Tiere.1972b; 73:95-116.

16. Hashimoto Y. Phylogenetic study of Formicidae based on the sensillum structures of the antenna and mouthparts. Graduate school of science and technology. Kobe University, Kobe. 1990a; 95.

17. Renthal R, Velasqueza D, Olmosa D, Hamptona J, Wergin WP. Structure and distribution of antennal sensilla of red imported fire ant. Micron 2003; 34:405-413.

18. Barsagade DD, Tembhare DB, Kadu SG. Microscopic structure of antennal sensilla in the carpenter ant Camponotus compressus (Fabricius) (Formicidae: Hymenoptera). Asian Myrmicology 2013; 5:113-120.

19. Mysore K, Shyamala BV, Rodrigues V. Morphological and developmental analysis of peripheral antennal chemosensory sensilla and central olfactory glomeruli in worker caste of Componotus compressus (Fabricius, 1787). Arthropod Structure and Development 2010; 39:310-321.

20. Nakanishi A, Nishino H, Watanbe H, Yokohari F, Nishikawa M. Sexspecific antennal sensory system in the ant Componotus japoincus: glomerural organizations of antennal lobes. Journal of Comparative Neurology 2010; 518:2186-2201.

21. Dumpert K. Alarmstoffrezeptoren auf der Antenne von Lasius fuliginosus (Hymenoptera: Formicidae). Zeitschrift Morphologie der Tiere 1972a; 73:95-116.

22. Zhou CX, Sun X, Mi F, Chen J, Wang MQ. Antennal sensilla in the parasitoid Scleroderma $s p$. (Hymenoptera: Bethylidae). Journalof Insect Science 2015; 15(36).

23. Hashimoto Y. Unique features of sensilla on the antennae of Formicidae (Hymenoptera). Applied Entomology and Zoology 1990b; 25:491-501.

24. Masson C. Quelues donnees sur l'ultrastructure de recpteurs gustatifs de l'antenne de la fourmi Camponotus vagus Scop. (Hymenoptera, formicinae). Z morphol. Tiere 1974; 77:235-243.

25. Villa L, Mineo G. Morphology of antennal structures of Grysonboseli Mineo and Szabo (Hymenoptera: Scelionidae): A scanning electron microscope study. Frustula Entomol. s. n. 1990a; 13:9-17.

26. Villa L, Mineo G. Mapping of the antennal structures of Grysonpennsylvanicum (Ashmead): A SEM study (Hymenoptera: Scelionidae). Frustula Entomol. s. n. 1990b; 13:225-235.

27. Rutchy M, Romani R, Kuebler LS, Ruschioni S, Roces F, Isidoro N, Kleineidam CJ. The thermo-sensitive sensilla coeloconica of leafcutting ants (Atta vollenwenderi). Arthropod Structure and Devlopment 2009; 38:195-205.

28. Nishino H, Nishikawa M, Mizunami M, Yokohari F. Functional and 
topographic segregation of glomeruli revealed by local staining of antennal sensory neurons in the honeybee Apis mellifera. Journal of Comparative Neurology 2009; 515:161-180.

29. Yokohari F, Tominaga Y, Tateda H. Antennal hygroreceptors of the honey bee, Apis mellifera L. Cell Tissue Research 1982; 226:63-73.

30. Drilling K, Klass KD. Surface structures of the antenna of mantophasmatodea (Insecta). Zoologisch. Anzeig A Journal of Comparative Zoology 2010; 249:121-137.

31. Giglio A, Brandmayr P, Ferrero EA, Perrota E, Romeo M, Zetto T, Talarico F. 2008. Comparative antennal morphometry and sensilla distribution pattern of three species of Siagoninae (Coleoptera, Carabidae). In: Penev L, Erwin T, Assmann T. (Editors). Back to the roots and back to the future: Towards a new synthesis amongst taxonomic ecological and biogeographical approaches in carbidology.
Sofia-moscow: Prnsoft, p. 143-158.

32. Yokohari F. The coelocapitular ssensillum, an antennal hygro- and thermo-receptive sensillum of the honey bee, Apis mellifera L. Cell Tissue Research 1983; 233:355-365.

33. Altner H, Prillinger L. Ultrastructure of invertebrate chemo-, thermo-, and hygroreceptors and its functional significance. International Review of Cytology 1980; 67:69-139.

How to cite this article:

Barsagade DD, Nagarkar DA, Kirsan JR. Surface Ultrastructure of Antenna and Distribution of Sensilla in the Leptogenys chinensis (Mayr) (Fabricus) (Formicidae: Hymenoptera). J App Biol Biotech. 2019;7(2):1- 6. DOI: 10.7324/JABB.2019.70201 